\title{
Decreased CRHBP expression is predictive of poor prognosis in patients with hepatocellular carcinoma
}

\author{
HAI-BING XIA ${ }^{1-3^{*}}$, HUI-JU WANG ${ }^{2,3^{*}}$, LUO-QIN FU ${ }^{2,3}$, SHI-BING WANG ${ }^{2,3}$, LI LI $^{2,3}$, GUO-QING RU ${ }^{2-4}$, \\ XIANG-LEI HE ${ }^{2-4}$, XIANG-MIN TONG ${ }^{2,3}$, XIAO-ZHOU MOU ${ }^{2,3}$ and DONG-SHENG HUANG ${ }^{2,3}$ \\ ${ }^{1}$ Clinical Department of Graduate School, Bengbu Medical College, Bengbu, Anhui 233003; \\ ${ }^{2}$ Clinical Research Institute, Zhejiang Provincial People's Hospital, People's Hospital of Hangzhou Medical College; \\ ${ }^{3}$ Key Laboratory of Tumor Molecular Diagnosis and Individualized Medicine of Zhejiang; ${ }^{4}$ Department of \\ Pathology, Zhejiang Provincial People's Hospital, Hangzhou, Zhejiang 310014, P.R. China
}

Received October 26, 2017; Accepted April 11, 2018

DOI: $10.3892 / \mathrm{ol} .2018 .9073$

\begin{abstract}
Corticotropin releasing hormone binding protein (CRHBP) mediates the reaction between corticotropin releasing hormone $(\mathrm{CRH})$ and corticotropin releasing hormone receptors (CRHRs). It is expressed in a number of organs, and the expression of CRHBP is associated with tumorigenesis and cancer progression. The aim of the present study was to investigate CRHBP expression levels in hepatocellular carcinoma (HCC) and its association with patient clinicopathological characteristics as well as prognosis. The expression of CRHBP was examined by immunohistochemistry in $169 \mathrm{HCC}$ tissues and 151 adjacent non-tumorous tissues. The results were validated by western blotting using patient tissues and liver cancer cell lines. The association of CRHBP expression with clinicopathological patient characteristics and survival rate was analyzed statistically. Expression of CRHBP was detected in 142/151 (94.0\%) non-tumorous liver tissues, and 84/169 (49.7\%) HCC tissues $(\mathrm{P}<0.001)$. The expression of CRHBP was negatively associated with tumor size $(\mathrm{P}=0.013)$, Edmondson Grade $(\mathrm{P}=0.002)$, hepatitis $B$ virus antigen $(\mathrm{P}=0.020)$, and $\alpha$-fetoprotein levels $(\mathrm{P}=0.014)$. Patients exhibiting low CRHBP expression were associated with shorter survival time compared with those exhibiting high CRHBP expression $(\mathrm{P}=0.012)$. The results of western blotting analysis suggest that reduced CRHBP expression is frequently observable in patients with HCC.
\end{abstract}

Correspondence to: Dr Dong-Sheng Huang, Clinical Research Institute, Zhejiang Provincial People's Hospital, People's Hospital of Hangzhou Medical College, 158 Shangtang Road, Hangzhou, Zhejiang 310014, P.R. China

E-mail: dshuang@zju.edu.cn

*Contributed equally

Key words: corticotropin releasing hormone binding protein, hepatocellular carcinoma, immunohistochemistry, bioinformatics analysis
Low CRHBP expression in HCC tissues may be a predictor of clinical prognosis and a potential therapeutic target for HCC.

\section{Introduction}

Hepatocellular carcinoma (HCC) is one of the most common types of malignancies, and ranks as the fifth most prevalent cancer worldwide $(1,2)$. Tumorigenesis is a complicated process, physical, chemical, and biological factors could lead to mutations in genes and the formation of tumors (3). HCC is common in China with an incidence rate of $250.28 / 100,000$ and accounts for $>50 \%$ of cancer cases worldwide $(4,5)$, which causes a heavy economic and physical burden to patients. Despite progress in conventional therapies, including surgical resection, percutaneous ablation, chemotherapy and embolization treatment, the 5-year survival rate of patients with HCC remains poor (6). Tumors have the characteristic of metastasis, which impedes of the ability of complete surgical resection, and recurrence is the biggest obstacle for the treatment of tumors (7). This suggests that diagnosis of HCC at an early stage is crucial for effective therapy and good prognosis, and highlights the urgent requirement for identification of biomarkers of HCC.

Corticotropin releasing hormone binding protein (CRHBP) is a 37-kDa oligopeptide, and is a component of the hypothalamic-pituitary-adrenal axis, involved in regulation of physiological reactions (8). Expression of CRHBP has been detected in numerous tissue types $(9,10)$. CRHBP prevents inappropriate activation of corticotropin releasing hormone (CRH) by binding the CRH complex (11).

CRHBP has been investigated in the field of normal physiologic, metabolic function and oncogenesis $(12,13)$. It was reported that Caucasians and African Americans with low CRHBP expression were associated with an increased risk of breast cancer (14). Low CRHBP mRNA and protein expression has been reported in prostatic and bladder cell carcinoma $(15,16)$.

Because the study of CRHBP expression in HCC tissue is unclear, the present study used immunohistochemistry, western blotting and reverse transcription-quantitative 
polymerase chain reaction (RT-qPCR) and bioinformatics analysis to evaluate the expression level of CRHBP in HCC. The association between CRHBP expression and clinicopathology and overall survival time was also investigated.

\section{Materials and methods}

Patients and samples. A total of 169 tumor tissue samples and 151 adjacent non-tumorous liver tissue samples were collected at surgery from patients with $\mathrm{HCC}$ at Zhejiang Provincial People's Hospital (Zhejiang, China) between January 2010 and December 2016. The patient cohort included 142 males and 27 females, with an age range of 25-90 years old and a mean age of 58 years old. All tissues were fixed with $4 \%$ formalin for $24 \mathrm{~h}$ at room temperature, paraffin-embedded, and diagnosis was confirmed by pathologists at Zhejiang Provincial People's Hospital (Zhejiang, China). Information regarding tumor size, number, location, Edmondson Grade and tumor metastasis were collected from patient medical records. Overall survival (OS) was defined as the time from the date of surgery to the date of mortality or the last day of follow up. The research was approved by the Zhejiang Provincial People's Hospital Ethics Committee (Zhejiang, China), and informed consent was obtained from each participant.

Immunohistochemical staining. Using the paraffin-embedded specimens, two tissue microarrays were designed and constructed by BioChip Company (Shanghai, China, www.shbiochip.com). Briefly, two tissue microarrays in $\left(5-\mu \mathrm{m}\right.$ thick) were incubated at $70^{\circ} \mathrm{C}$ for $2 \mathrm{~h}$, then deparaffinized in xylene $10 \mathrm{~min}$ for three times, prior to rehydration in a graded ethanol series $(100,95,85$ and $75 \%$ for $5 \mathrm{~min})$. For antigen retrieval, the microarrays were boiled at $120^{\circ} \mathrm{C}$ with TE buffer (1.21 g/l Tris, $0.37 \mathrm{~g} / 1$ EDTA, $0.5 \mathrm{ml} / 1$ Tween-20) at high pressure for $3 \mathrm{~min}$. The sections were then treated with $3 \%$ hydrogen peroxide for $15 \mathrm{~min}$ at room temperature to eliminate endogenous peroxidase activity, and blocked with $10 \%$ goat serum (reagent A; Histostain-plus Bulk kit; Thermo Fisher Scientific, Inc., Waltham, MA, USA) at room temperature for $20 \mathrm{~min}$. The sections were then incubated with a CRHBP primary antibody (dilution, 1:200, AF2796; R\&D Systems, Inc., Minneapolis, MN, USA) overnight at $4^{\circ} \mathrm{C}$. Following 3 washes with PBS, the sections were incubated with a biotinylated second antibody (reagent B; Histostain-plus Bulk kit) for 15 min, prior to the addition of streptavidin-peroxidase (reagent C, Histostain-plus Bulk kit) for 15 min at room temperature, according to the manufacturer's protocol. Finally, a chromogenic reaction was performed using a DAB color-substrate solution (OriGene Technologies, Inc., Rockville, MD, USA), according to the manufacturer's protocol. Color development was stopped when the brown was observed obviously in the tissue microarrays. Hematoxylin (cat. no. C0107; Beyotime Institute of Biotechnology, Haimen, China) staining was performed for 3-5 min. The process was completed with dehydration in $75,85,95$, and $100 \%$ ethyl alcohol for $5 \mathrm{~min}$ respectively, transparency three times in xylene for $10 \mathrm{~min}$ and mounting with gelatin resin. All procedures were performed at room temperature unless otherwise specified.
Evaluation of the immunohistochemistry. The results of immunohistochemical CRHBP staining were interpreted by 2 pathologists of the Pathology Department of Zhejiang Provincial People's Hospital, considering the staining intensity and the proportion of stained cells. The staining intensity was scored as follows: 0 , negative staining; 1 , weak staining; 2 , medium staining, and 3 , strong staining. The proportion of stained cells were scored as follows: 0 , no cells stained; 1 , $1-25 \%$ cells stained; $2,26-50 \%$ cells stained; $3,51-75 \%$ cells stained, and $4,>75 \%$ cells stained. We multiplied the staining intensity and proportion scores to confirm the level of CRHBP expression: 0 was considered to indicate negative expression, and $\geq 1$ was considered to indicate positive expression.

Cell culture. Human liver cancer cell lines HCCLM3, MHCC97H, Huh7, Hep3B, HepG2 were obtained from cell bank of Shanghai Academy of Sciences (Shanghai, China), and cultured in DMEM (SH30243.01; HyClone; GE Healthcare Life Sciences, Logan, UT, USA) containing $10 \%$ fetal bovine serum (16000-044; Gibco; Thermo Fisher Scientific, Inc., Waltham, MA, USA), $1 \%$ penicillin-streptomycin solution (SV30010; Hyclone). All cells were maintained in a humidified incubator with $5 \% \mathrm{CO}_{2}$ at $37^{\circ} \mathrm{C}$.

Western blot analysis. CRHBP expression was analyzed in three paired $\mathrm{HCC}$ and adjacent non-cancerous tissues and five liver cancer cell lines (HCCLM3, MHCC97H, Huh7, Hep3B, HepG2). Proteins were extracted with RIPA buffer (cat. no. P0013B; Beyotime Institute of Biotechnology) and concentration determined by BCA Kit (cat. no. P0009; Beyotime Institute of Biotechnology). Then, proteins were heat-inactivated at $100^{\circ} \mathrm{C} 10 \mathrm{~min}, 12 \%$ SDS-PAGE $(20 \mu \mathrm{g}$ protein/lane) and transferred to polyvinylidene fluoride membranes by electrophoresis. The membranes were blocked in 5\% skim milk for $1.5 \mathrm{~h}$ at room temperature. A CRHBP primary antibody (dilution, 1:1,000, AF2796; R\&D) was incubated with the membranes overnight at $4^{\circ} \mathrm{C}$. The membranes were washed in Tris-buffered saline with Tween three times prior to addition of a horseradish peroxidase conjugated-goat anti-Mouse IgG secondary antibody (dilution, 1:5,000, HA1006; HuaAn Biotechnology Co., Ltd., Hangzhou, China) for $1.5 \mathrm{~h}$ at room temperature. Finally, protein expression levels were analyzed via grey level was using Quantity One software (version 4.6.2; Bio-Rad Laboratories, Inc., Hercules, CA, USA).

RT-qPCR analysis. Total RNA was isolated from five liver cancer cell lines (HCCLM3, MHCC97H, Huh7, Hep3B, HepG2) using TRIzol Reagent (Invitrogen; Thermo Fisher Scientific, Inc.) according to the manufacturer's protocol. cDNAs were synthesized from $1 \mu \mathrm{g}$ of DNase-treated total RNA using the PrimeScriptTM RT reagent kit with gDNA Eraser (Takara Bio, Inc., Otsu, Japan), under $65^{\circ} \mathrm{C}, 5 \mathrm{~min}, 42^{\circ} \mathrm{C}, 60 \mathrm{~min}$ and $70^{\circ} \mathrm{C}$, 10 min of reverse transcription. The primers of CRHBP were 5'-CACACCAGCATCGAAACTGC-3' (forward) and 5'-TGA AGACCATTTACGTGTCCCA-3' (reverse). The Primers for GAPDH were 5'-TGAAGGTCGGAGTCAACGG-3' (forward) and 5'-CTGGAAGATGGTGATGGGATT-3' (reverse). qPCR was carried out using a Bio-Rad CFX Connect Real-Time system (Bio-Rad Laboratories, Inc) using the thermal cycling condition: $98^{\circ} \mathrm{C}$ for 2 min followed by 39 amplification 
Table I. Associations between expression of CRHBP protein and clinicopathological characteristics in 169 cases of hepatocellular carcinoma.

\begin{tabular}{lll}
\hline & CRHBP expression \\
Clinical parameters Number & Negative Positive P-value
\end{tabular}

\begin{tabular}{lrll}
\hline Age (years) & & & \\
$\geq 55$ & 107 & 53 & 5 \\
$<55$ & 62 & 32 & 30 \\
Sex & & & \\
Male & 142 & 71 & 71 \\
Female & 27 & 14 & 13
\end{tabular}

Tumor diameter (cm)

$<5$

$$
99
$$

Tumor number

Single
Multiple
Location
Left
Right
Left + right
Edmondson
I+II
III
Metastasis
M0
M1

Microvascular

invasion

\begin{tabular}{lrrrr} 
Absent & 70 & 35 & 35 & \\
Present & 59 & 34 & 25 & \\
HBV antigen & & & & 0.020 \\
Negative & 34 & 23 & 11 & \\
$\quad$ Positive & 130 & 60 & 70 & \\
Cirrhosis & & & & 0.217 \\
Negative & 52 & 29 & 23 & \\
Positive & 117 & 56 & 61 & \\
AFP (ng/ml) & & & & 0.014 \\
$<50$ & 91 & 36 & 55 & \\
$\geq 50$ & 78 & 45 & 33 & \\
Survival status at & & & & 0.009 \\
end of follow-up & & & & \\
Not alive & 41 & 27 & 14 & \\
Alive & 84 & 35 & 49 & \\
\hline
\end{tabular}

CRHBP, corticotropin releasing hormone binding protein; M0, no metastasis identified; M1, metastasis identified; HBV, hepatitis B virus; AFP, $\alpha$-fetoprotein

cycles at $98^{\circ} \mathrm{C}$ for $15 \mathrm{sec}$ and $60^{\circ} \mathrm{C}$ for $15 \mathrm{sec}$. At the end of the PCR cycles, melting curve analysis were performed. The
Table II. Expression of CRHBP protein in HCC and normal liver tissues.

\begin{tabular}{lcccc}
\hline & & \multicolumn{2}{c}{ CRHBP expression } & \\
\cline { 3 - 4 } Tissue type & Number & Negative & Positive & P-value \\
\hline HCC & 169 & 85 & 84 & $\mathrm{P}<0.001$ \\
$\begin{array}{l}\text { Adjacent } \\
\text { non-tumorous }\end{array}$ & 151 & 9 & 142 & \\
\hline
\end{tabular}

HCC, hepatocellular carcinoma; CRHBP, corticotropin releasing hormone binding protein.

expression of CRHBP was normalized to GAPDH using $2^{-\Delta \Delta C \mathrm{C}}$ method (17).

Bioinformatics analysis using online databases and search tool for the retrieval of interacting genes/proteins. CRHBP mRNA expression in HCC and normal tissues was compared using the online Oncomine database (https://www.oncomine.org) with the following filtering conditions: Gene, CRHBP; analysis type, cancer vs. normal; cancer type, hepatocellular carcinoma; data type, mRNA. The 10-year survival analysis was performed using OncoLnc (http://www.oncolnc.org/). Gene enrichment analysis of CRH was performed using STRING (https://string-db.org/) as there was not enough CRHBP data in the online database, and the top 20 enriched genes analyzed to investigate its biological function. Target signaling pathway was searched on KEGG database (www.genome.jp).

Statistical analysis. Statistical analysis was performed using SPSS software (version 16.0; SPSS, Inc., Chicago, IL,USA). $\chi^{2}$ test was used to analyze the association between CRHBP expression and clinicopathological parameters. The overall survival curve was generated using the Kaplan-Meier method and further analyzed using the log-rank test. $\mathrm{P}<0.05$ was considered to indicate a statistically significant difference.

\section{Results}

Clinical characteristics of patients. The majority of the patients who participated in the present study had a history of hepatitis B virus infection (79.3\%). Upon the last day of follow-up, 84 patients were alive and 41 mortalities had occurred. A total of 44 patients were lost to follow-up (Table I).

CRHBP expression in HCC. Positive immunohistochemical staining of CRHBP was detected in 84/169 (49.7\%) HCC tissues and 142/151 (94.0\%) adjacent non-tumorous tissues. The expression of CRHBP in tumor tissues was significantly lower than that in adjacent non-tumorous tissues $(\mathrm{P}<0.001$; Table II; Fig. 1A and B). A bar chart illustrating the score values of the immunostaining of CRHBP expression in HCC tissues (Fig. 1C).

The association between CRHBP expression and clinicopathological characteristics. Tumor diameters $\geq 5 \mathrm{~cm}$ were significantly associated with low expression of CRHBP 
A

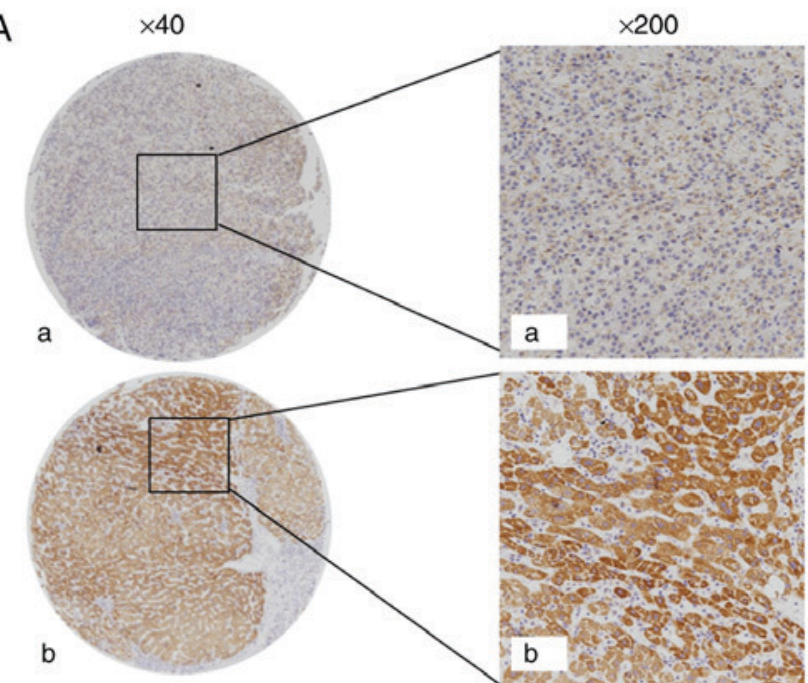

B

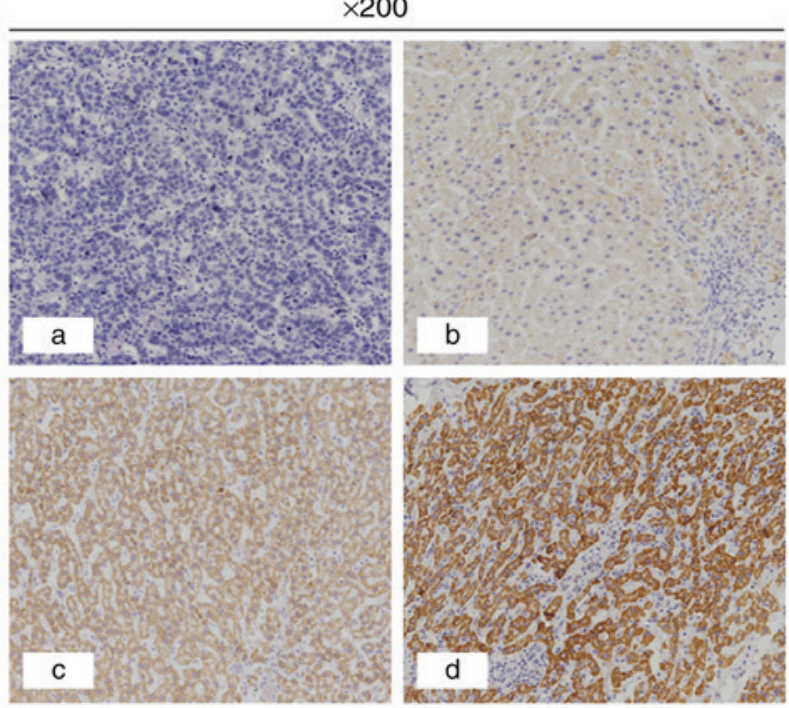

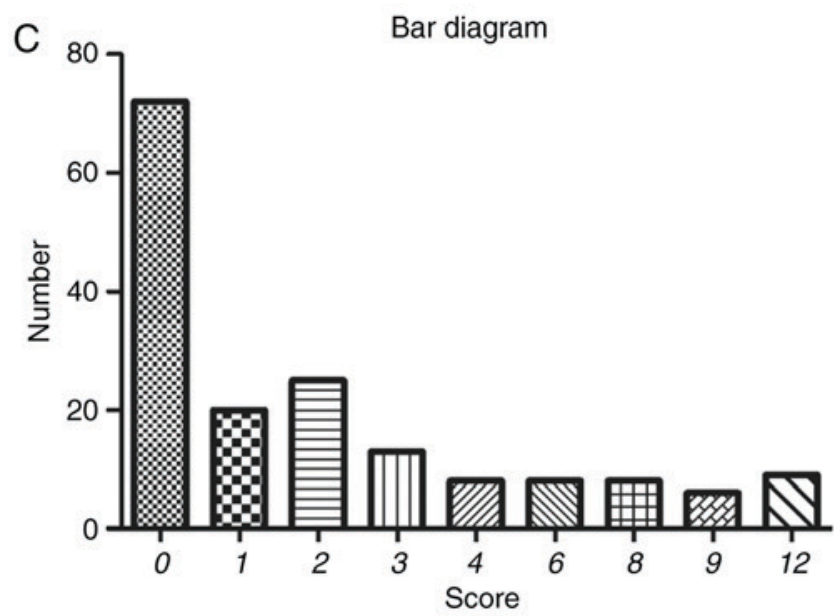

Figure 1. CRHBP protein expression in tumor and adjacent non-cancerous tissues. (A) a) Low expression of CRHBP in tumor specimens; b) high expression of CRHBP in paired adjacent tissues. Magnification x200 or x40, as indicated. (Ba) Negative (-); (Bb) positive (+); (Bc) positive (++), and, (Bd) positive (+++) immunohistochemical staining of CRHBP in HCC tissues. Magnification, x200. (C) A bar chart representing the immunostaining scores of CRHBP protein expression in HCC tissues. CRHBP, corticotropin releasing hormone binding protein; HCC, hepatocellular carcinoma.

$(\mathrm{P}=0.013)$. Low CRHPB expression was also significantly associated with Edmondson Grade ( $\mathrm{P}=0.002)$, high $\alpha$-fetoprotein (AFP) levels $(\mathrm{P}=0.014)$ and hepatitis $\mathrm{B}$ virus (HBV) infection $(\mathrm{P}=0.020)$. No significant association was identified between CRHBP expression and age, sex, tumor location, metastasis status, microvascular invasion or cirrhosis $(\mathrm{P}>0.05$; Table I).

Association between CRHBP expression and prognosis. To evaluate the prognostic value of CRHBP expression, Kaplan-Meier curves were constructed. The 5-year overall survival rate was significantly different between patients exhibiting low CRHBP expression (39.20 months) and patients exhibiting high CRHBP expression (49.18 months) ( $\mathrm{P}=0.012$; log-rank test; Fig. 2). Thus, it was inferred that patients exhibiting low CRHBP expression in the liver tissue had a reduced survival time compared with those exhibiting high CRHBP expression.

CRHBP is downregulated in liver cancer tissues and cell lines. To further confirm the expression pattern of

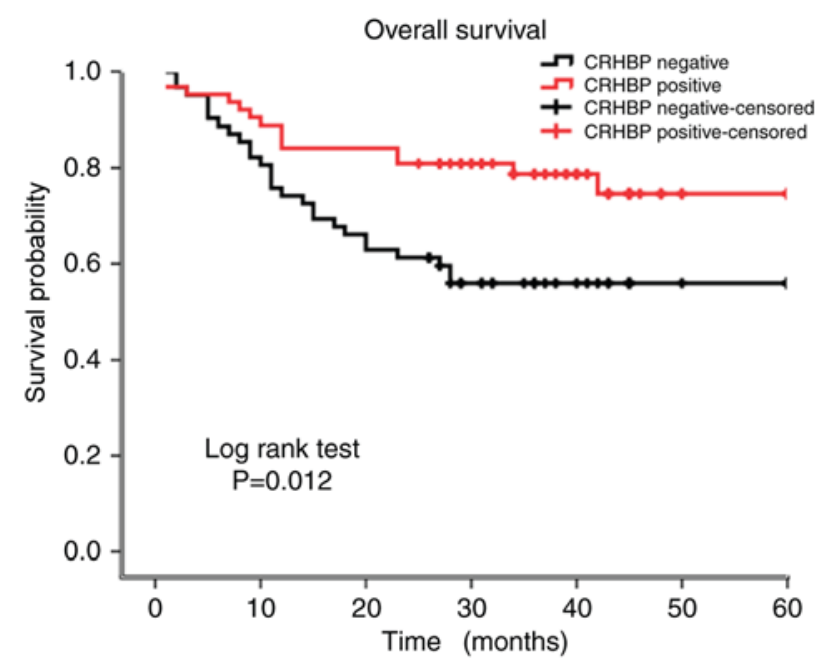

Figure 2. Kaplan-Meier survival curves for patients with HCC exhibiting high or low CRHBP expression levels. Patients exhibiting low CRHBP expression level were associated with a significantly shorter survival time than those exhibiting high CRHBP expression. HCC, hepatocellular carcinoma; CRHBP, corticotropin releasing hormone binding protein. 

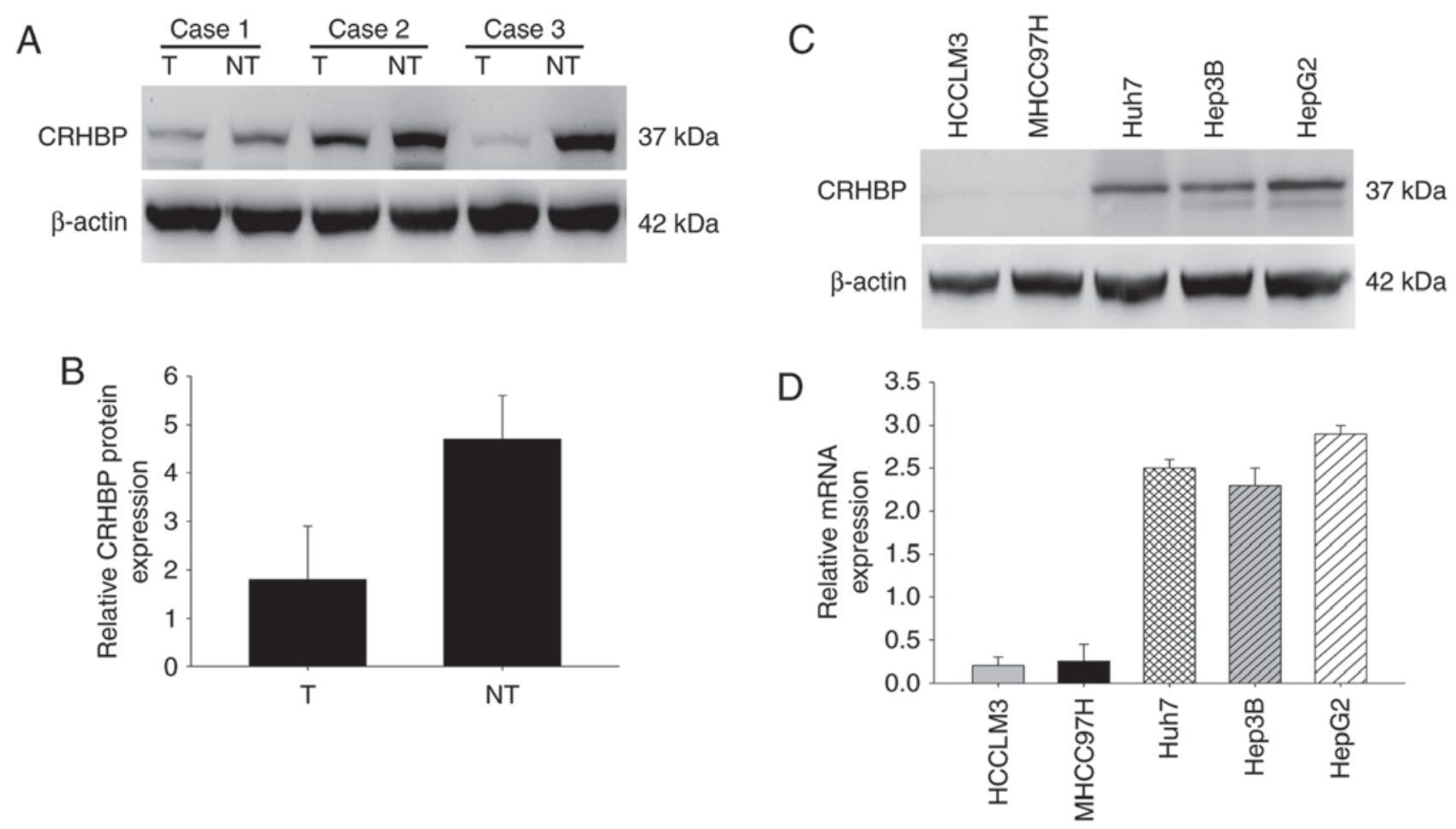

Figure 3. CRHBP expression levels in HCC tissues determined by western blotting and reverse transcription-quantitative polymerase chain reaction. (A) CRHBP protein expression levels in 3 paired $\mathrm{T}$ and adjacent NT tissues. (B) A low expression level of CRHBP protein was detected in HCC tumor tissues compared with that in adjacent non-tumorous tissues. (C) Western blot analysis of CRHBP expression in liver cancer cell lines. (D) mRNA expression level of CRHBP was detected by reverse transcription-quantitative polymerase chain reaction (mean \pm standard deviation). CRHBP, corticotropin releasing hormone binding protein; HCC, hepatocellular carcinoma; T, tumor tissue; NT, non-tumorous tissue.

CRHBP in HCC, the protein expression level of CRHBP was determined in paired HCC and adjacent non-cancerous liver tissues by western blotting. Compared with the adjacent non-tumor tissues, significant downregulation of CRHBP protein expression was observed in HCC tissues (Fig. 3A and B). Furthermore, CRHBP levels were lower in highly metastatic liver cancer cell lines, including HCCLM3 and MHCC97H cells, than in those with low metastatic potential, including Huh7 and Hep3B cells, and was highest in HepG2 hepatic cancer cells as determined by western blotting and RT-qPCR (Fig. 3C and D). These results suggest that reduced CRHBP expression is common in patients with HCC, and that this may be associated with tumorigenesis.

Bioinformatics analysis of CRHBP in patients with HCC. A total of 5 datasets: Wurmbach Liver Statistics (18), Chen Liver Statistics (19), Roessler Liver Statistics, Roessler Liver 2 Statistics (20) and Mas Liver Statistics (21), were selected to analyze the expression of CRHBP in HCC vs. normal tissues using Oncomine databases. It was demonstrated that the expression of CRHBP mRNA was significantly lower in HCC tissues than in normal tissues (Fig. 4; $\mathrm{P}<0.01$ ). OncoLnc was used to reveal that patients exhibiting low expression of CRHBP had a shorter 10-year survival time (Fig. 5; log-rank, $\mathrm{P}=0.0122$ ). STRING was used to identify the top 20 biological processes of $\mathrm{CRH}$ gene enrichment (Table III) and a part of an important signaling pathway of cAMP was generated on KEGG database (Fig. 6).

\section{Discussion}

Oncogenesis is a multi-factorial process, and numerous genes have been identified to be involved in HCC oncogenesis (22). However, the role of CRHBP expression in HCC remains unclear. The aim of the present study was to investigate the association between CRHBP expression and HCC.

Low expression of CRHBP was associated with large tumor size $(\mathrm{P}=0.013)$ and high Edmondson Grade $(\mathrm{P}=0.002)$. This supports previous research in human kidney cancer which suggested that low expression of CRHBP in kidney tumor cell lines could promote proliferation (16). In the present study, microvascular invasion and metastasis had no association with CRHBP expression; however, Tezval et al (15) reported that clear cell renal cell cancer with low expression of CRHBP had strong invasion and metastasis. This may be due to the different sources of the tissues or the change from in vivo to ex vivo microenvironment. Besides, as CRHBP has a high affinity for CRH and urocortin (23), we could infer that when CRHBP expression was downregulated, the stimulate signal from CRH would be decreased, and the physiological of the hepatocyte might be changed. As it has been previously reported that $\mathrm{CRH}$ promotes the proliferation of human colon cancer cells and upregulates VEGF expression (24). Gene enrichment analysis of the biological processes associated with $\mathrm{CRH}$ indicated that $\mathrm{CRH}$ may regulate cytobiological state via the cAMP signaling pathway. We hypothesize that CRHBP may prevent CRH from activating CRHRs in incorrect locations or in excess. 
A

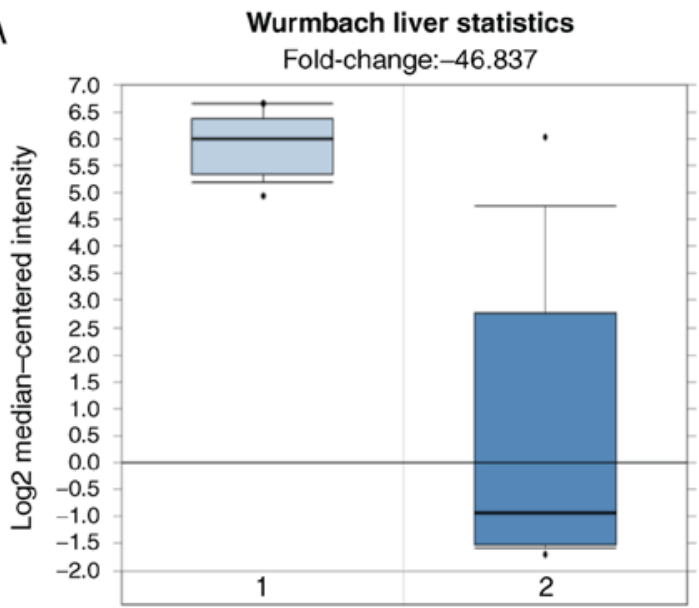

1. Liver (10)

2. Hepatocellular carcinoma (35)

C

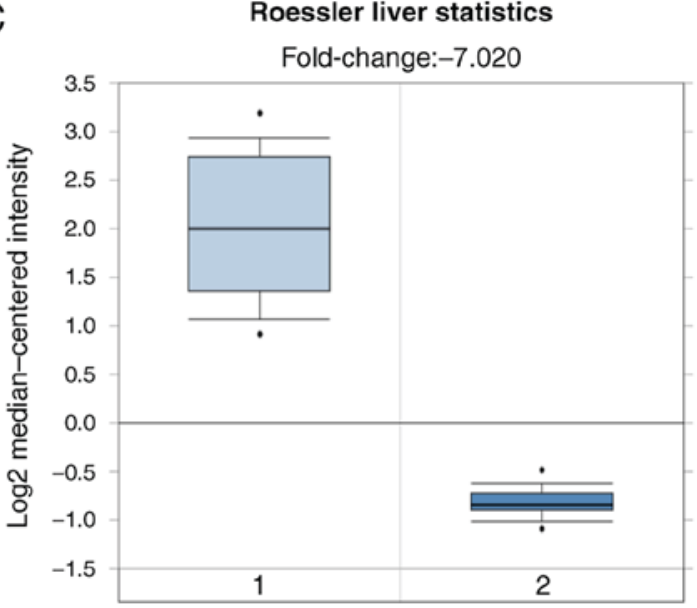

1. Liver (21)

2. Hepatocellular carcinoma (22)

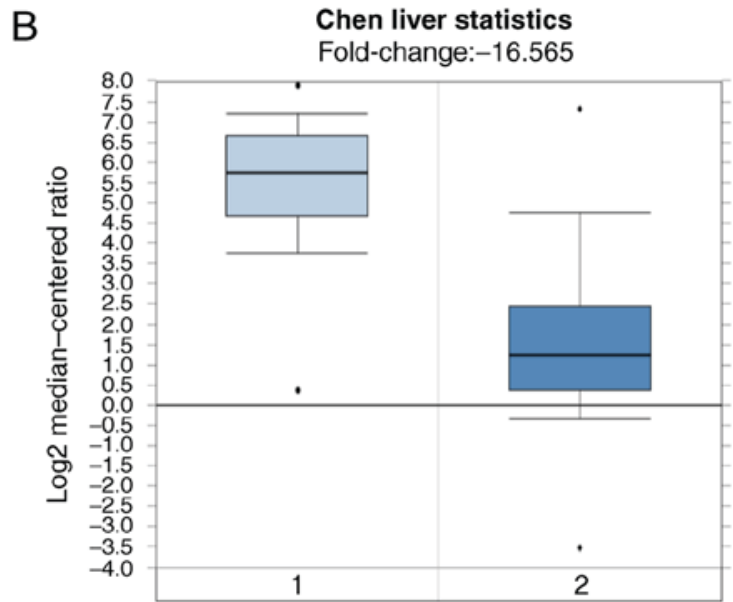

1. Liver (75)

2. Hepatocellular carcinoma (95)

D

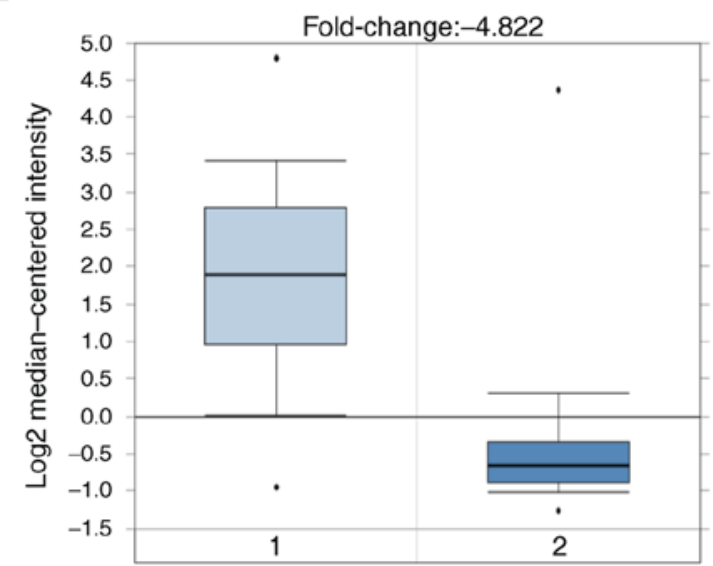

1. Liver (220)

2. Hepatocellular carcinoma (225)

E

Mas liver statistics

Fold-change:-1.817

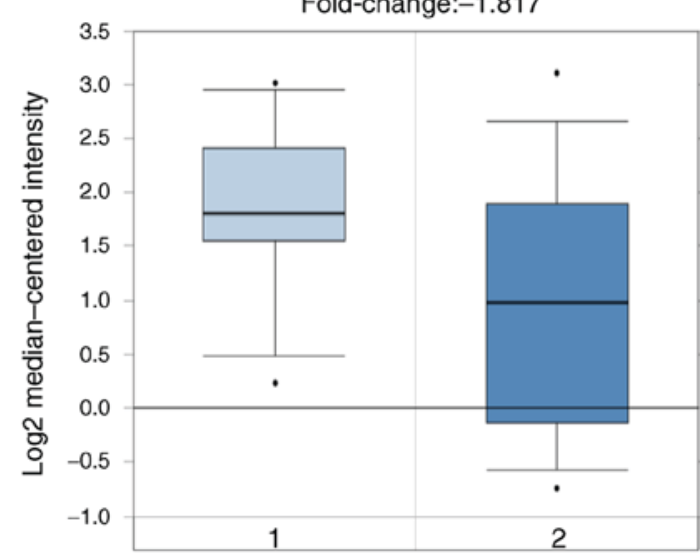

1. Liver (19)

2. Hepatocellular carcinoma (38)

Figure 4. Analysis of CRHBP expression in hepatocellular carcinoma vs. that in normal tissues using online Oncomine databases. (A-E) Graphs were obtained from the online Oncomine software. The mRNA expression of CRHBP was demonstrated to be significantly lower in HCC compared with normal liver tissue in all datasets. CRHBP, corticotropin releasing hormone binding protein; HCC, hepatocellular carcinoma.

Although HBV infection was demonstrated to be associated with CRHBP expression $(\mathrm{P}=0.020)$, the molecular mechanism behind downregulation of CRHBP following
HBV infection remains uncharacterized. Previous research suggests that CRBHP expression was decreased following long-term stimulation by $\operatorname{HBV}(13,25,26)$, and that 


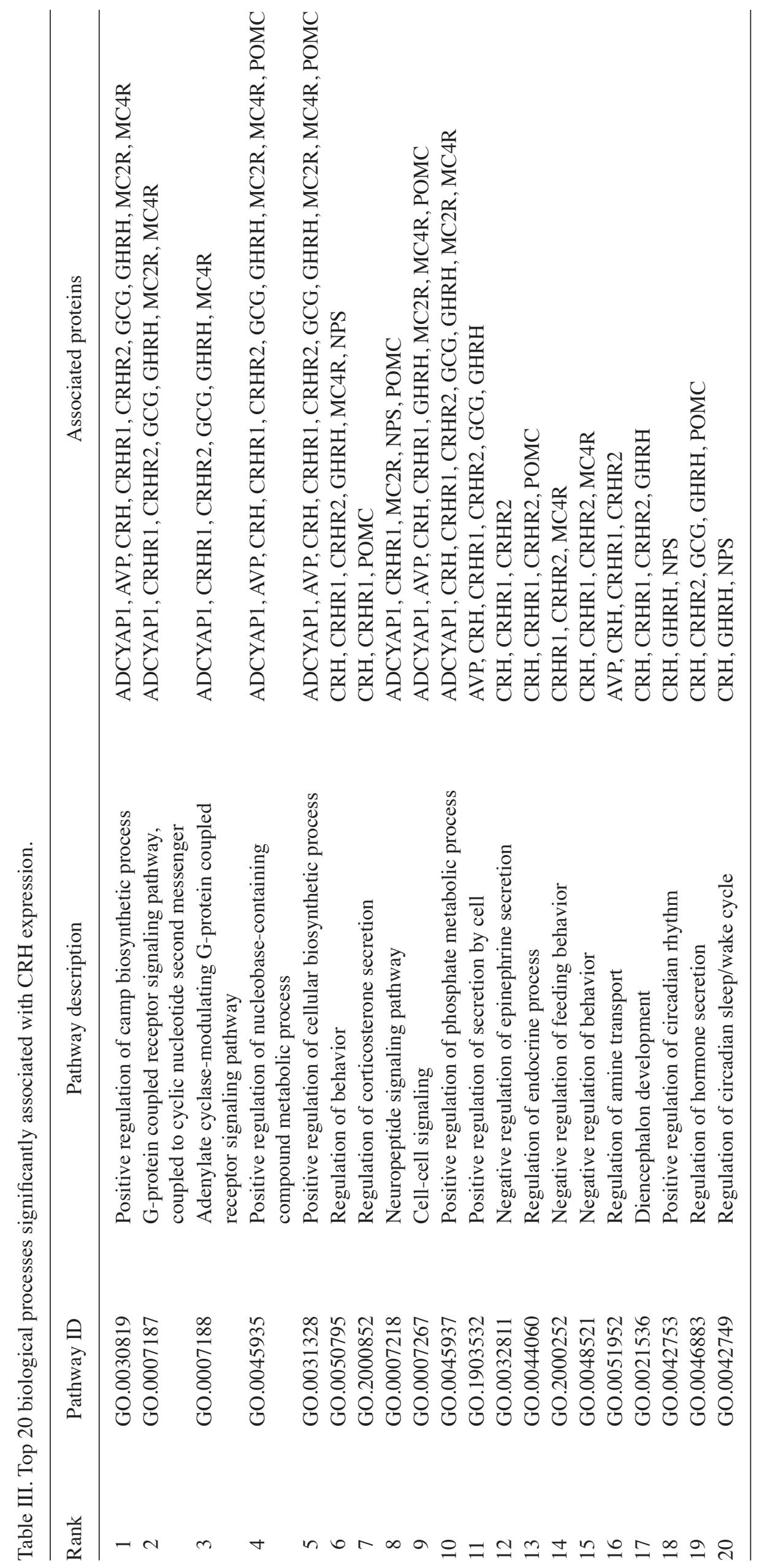




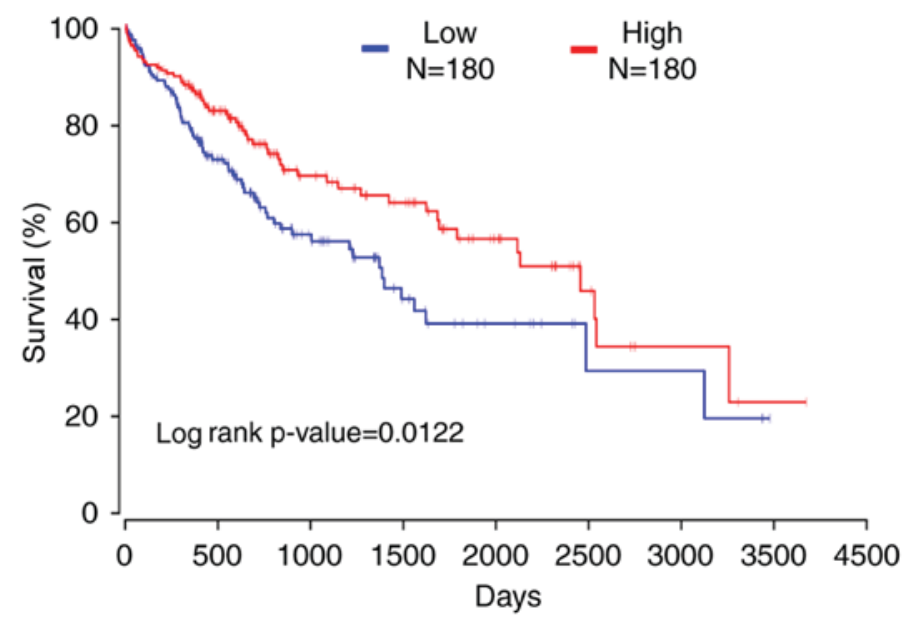

Figure 5. The 10-year survival rate of patients with hepatocellular carcinoma exhibiting high or low CRHBP expression. Patients exhibiting low CRHBP expression were associated with a significantly lower 10 -year survival rate. CRHBP, corticotropin releasing hormone binding protein; $\mathrm{N}$, number.

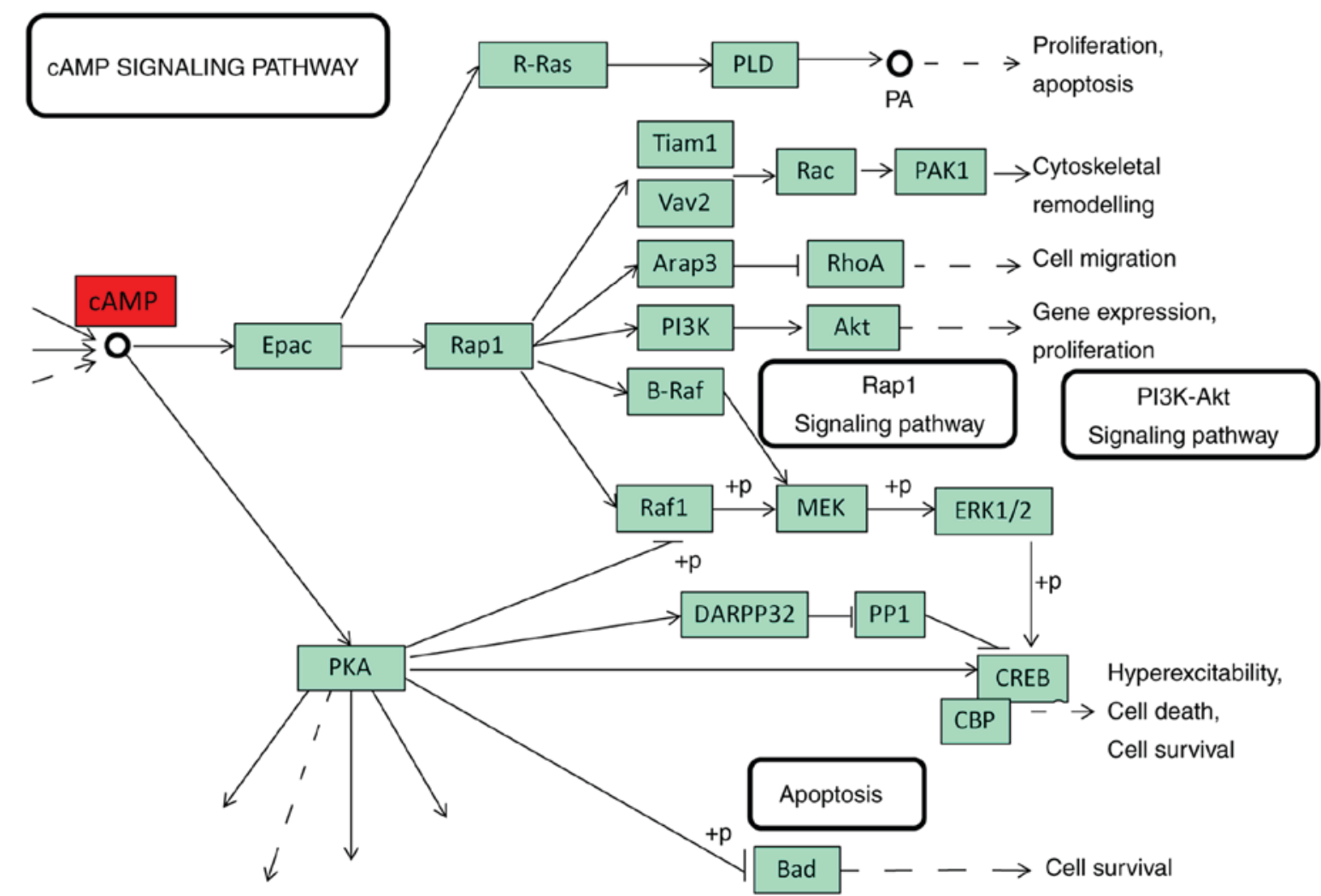

Figure 6. The cAMP pathway is associated with multiple biological processes, which were analyzed using Kyoto Encyclopaedia of Genes and Genomes. cAMP, cyclic AMP.

hypermethylation of the CRHBP gene caused its downregulation (15).

The results of the present study may have valuable clinical application. Upregulation of CRHBP expression may inhibit the proliferation of hepatocellular carcinoma cells as well as tumor progression. Clinicians should be aware of the significance of HBV infection when considering therapeutic strategies.

In conclusion, CRHBP expression was demonstrated to be associated with high AFP level and overall survival rate in $\mathrm{HCC}$, and may be a potential biomarker for HCC diagnosis, treatment and prognosis.

\section{Acknowledgements}

Not applicable.

\section{Funding}

This study was supported by the National Science Foundation of China (grant nos. 81672474, 81672430, 81602706 and 81602174), Zhejiang Provincial Natural Science Foundation of China (grant nos. LY15H160051, LQ16H160017 and LY16H160042), Funds of Science Technology Department of 
Zhejiang Province (grant no. 2016C33055) and the Zhejiang Province Bureau of Health (grant nos. WKJ-ZJ-1502 and 2015ZA009).

\section{Availability of data and materials}

The datasets used and/or analyzed during the current study are available from the corresponding author on reasonable request.

\section{Authors' contributions}

HX and HW wrote the manuscript and conducted the experiments; LF, SW and LL performed data analysis. GR and XH were in charge of sample processing. XM designed the present study and revised the manuscript. XT and DH collected clinical data and provided funding.

\section{Ethics approval and consent to participate}

The research was approved by Review Board of Hospital Ethics Committee, and the informed consent from each participant was obtained before data collection.

\section{Patient consent for publication}

Written informed consent was obtained from the patient for the publication of this paper together with the accompanying images.

\section{Competing interests}

The authors declare that they have no competing interests.

\section{References}

1. Jemal A, Bray F, Center MM, Ferlay J, Ward E and Forman D: Global cancer statistics. CA Cancer J Clin 61: 69-90, 2011.

2. Torre LA, Bray F, Siegel RL, Ferlay J, Lortet-Tieulent J and Jemal A: Global cancer statistics, 2012. CA Cancer J Clin 65: 87-108, 2015.

3. Elsegood CL, Tirnitz-Parker JE, Olynyk JK and Yeoh GC: Immune checkpoint inhibition: Prospects for prevention and therapy of hepatocellular carcinoma. Clin Transl Immunol 6: e161, 2017.

4. Chen W, Zheng R, Baade PD, Zhang S, Zeng H, Bray F, Jemal A, Yu XQ and He J: Cancer statistics in China, 2015. CA Cancer J Clin 66: 115-132, 2016

5. Chen W, Zheng R, Zeng H, Zhang S and He J: Annual report on status of cancer in China, 2011. Chin J Cancer Res 27: 2-12, 2015.

6. Llovet JM and Bruix J: Systematic review of randomized trials for unresectable hepatocellular carcinoma: Chemoembolization improves survival. Hepatology 37: 429-442, 2003.

7. Chaffer CL and Weinberg RA: A perspective on cancer cell metastasis. Science 331: 1559-1564, 2011.

8. Ketchesin KD, Stinnett GS and Seasholtz AF: Corticotropin-releasing hormone-binding protein and stress: From invertebrates to humans. Stress 20: 449-464, 2017.

9. Behan DP, Linton EA and Lowry PJ: Isolation of the human plasma corticotrophin-releasing factor-binding protein. J Endocrinol 122: 23-31, 1989.

10. Peto CA, Arias C, Vale WW and Sawchenko PE: Ultrastructural localization of the corticotropin-releasing factor-binding protein in rat brain and pituitary. J Comp Neurol 413: 241-254, 1999.
11. Sutton SW, Behan DP, Lahrichi SL, Kaiser R, Corrigan A, Lowry P, Potter E, Perrin MH, Rivier J and Vale WW: Ligand requirements of the human corticotropin-releasing factor-binding protein. Endocrinology 136: 1097-1102, 1995.

12. Borowski KS, Clark EA, Lai Y, Wapner RJ, Sorokin Y, Peaceman AM, Iams JD, Leveno KJ, Harper M, Caritis SN, et al: Neonatal genetic variation in steroid metabolism and key respiratory function genes and perinatal outcomes in single and multiple courses of corticosteroids. Am J Perinatol 32: 1126-1132, 2015.

13. Kolasa M, Faron-Górecka A, Kuśmider M Szafran-Pilch K, Solich J, Żurawek D, Gruca P, Papp M and Dziedzicka-Wasylewska M: Differential stress response in rats subjected to chronic mild stress is accompanied by changes in $\mathrm{CRH}$-family gene expression at the pituitary level. Peptides 61: 98-106, 2014.

14. Nan H, Dorgan JF and Rebbeck TR: Genetic variants in hypothalamic-pituitary-adrenal axis genes and breast cancer risk in Caucasians and African Americans. Int J Mol Epidemiol Genet 6: 33-40, 2015.

15. Tezval H, Dubrowinskaja N, Peters I, Reese C, Serth K, Atschekzei F, Hennenlotter J, Stenzl A, Kuczyk MA and Serth J: Tumor specific epigenetic silencing of corticotropin releasing hormone-binding protein in renal cell carcinoma: Association of hypermethylation and metastasis. PLoS One 11: e0163873, 2016.

16. Tezval H, Atschekzei F, Peters I, Waalkes S, Hennenlotter J, Stenzl A, Becker JU, Merseburger AS, Kuczyk MA and Serth J: Reduced mRNA expression level of corticotropin-releasing hormone-binding protein is associated with aggressive human kidney cancer. BMC Cancer 13: 199, 2013.

17. Livak KJ and Schmittgen TD: Analysis of relative gene expression data using real-time quantitative PCR and the 2(-Delta Delta C(T)) method. Methods 25: 402-408, 2001.

18. Wurmbach E, Chen YB, Khitrov G, Zhang W, Roayaie S, Schwartz M, Fiel I, Thung S, Mazzaferro V, Bruix J, et al: Genome-wide molecular profiles of $\mathrm{HCV}$-induced dysplasia and hepatocellular carcinoma. Hepatology 45: 938-947, 2007.

19. Chen X, Cheung ST, So S, Fan ST, Barry C, Higgins J, Lai KM, Ji J, Dudoit S, Ng IO, et al: Gene expression patterns in human liver cancers. Mol Biol Cell 13: 1929-1939, 2002.

20. Roessler S, Jia HL, Budhu A, Forgues M, Ye QH, Lee JS, Thorgeirsson SS, Sun Z, Tang ZY, Qin LX and Wang XW: A unique metastasis gene signature enables prediction of tumor relapse in early-stage hepatocellular carcinoma patients. Cancer Res 70: 10202-10212, 2010.

21. Mas VR, Maluf DG, Archer KJ, Yanek K, Kong X, Kulik L, Freise CE, Olthoff KM, Ghobrial RM, McIver P and Fisher R: Genes involved in viral carcinogenesis and tumor initiation in hepatitis $C$ virus-induced hepatocellular carcinoma. Mol Med 15: 85-94, 2009.

22. Burkhart RA, Ronnekleiv-Kelly SM and Pawlik TM: Personalized therapy in hepatocellular carcinoma: Molecular markers of prognosis and therapeutic response. Surg Oncol 26: 138-145, 2017.

23. Inda C, Armando NG, Dos Santos Claro PA and Silberstein S: Endocrinology and the brain: Corticotropin-releasing hormone signaling. Endocr Connect 6: R99-R120, 2017.

24. Fang X, Hong Y, Dai L, Qian Y, Zhu C, Wu B and Li S: $\mathrm{CRH}$ promotes human colon cancer cell proliferation via IL-6/JAK2/STAT3 signaling pathway and VEGF-induced tumor angiogenesis. Mol Carcinog 56: 2434-2445, 2017.

25. Gouraud SS, Takagishi M, Kohsaka A, Maeda M and Waki H: Altered neurotrophic factors' expression profiles in the nucleus of the solitary tract of spontaneously hypertensive rats. Acta Physiol (Oxf) 216: 346-357, 2016.

26. Linnstaedt SD, Bortsov AV, Soward AC, Swor R, Peak DA, Jones J, Rathlev N, Lee DC, Domeier R, Hendry PL and McLean SA: CRHBP polymorphisms predict chronic pain development following motor vehicle collision. Pain 157: 273-279, 2016.

This work is licensed under a Creative Commons Attribution-NonCommercial-NoDerivatives 4.0 International (CC BY-NC-ND 4.0) License. 